Alzheimer's disea se is a serious concem in the aging population.

A substantial body of evidence exists to suggest that exc ess TNF- $\alpha$ plays a key role in the pathogenesis of Alzheimer's disea se

\title{
Case study of new drug suggests a novel method of targeting Alzheimer's symptoms
}

Etanercept $\left(\right.$ Enbrel $\left.^{\circledR}\right)$, currently licensed as a rheumatoid arthritis drug, is a biological antagonist of T N F- $\alpha$ and has been previously investigated as a potential treatment for Alzheimer's disease in a 6-month pilot study. A novel study, published online in January 9 in the Journal of $N$ euroinflammation, investigating the treatment has studied the effects of the drug on a single patient with late-onset Alzheimer's disease. The results demonstrated a rapid cognitive improvement in the patient following perispinal administration of etanercept.

\begin{tabular}{c}
\hline '...minutes after the \\
treatment was given $[. .$.$] he$ \\
a ppeared calmer, more \\
organized and \\
less frustrated.'
\end{tabular}

Testing the patient prior to administration of etanercept revealed poor cognitive performance; he was unable to relay the date, location or the name of the doctor treating him. H owever, minutes after the treatment was administered he was able to state where he was, give the date and perform simple arithmetic tasks that he had been previously unable to complete. The doctors noted that he appeared calmer, more organized and less frustrated.

Sue Griffin, editor of the Journal of $N$ euroinflammation and a researcher at the U niversity of Arkansas for M edical Sciences (AR, USA), who wrote an accompanying commentary, reported, "It is unprecedented to see cognitive and behavioural improvement in a patient with established dementia within minutes of therapeutic intervention. This gives all of us in Alzheimer research a tremendous new clue about new avenues of research." She added, "Even though this report predominantly discusses a single patient it is of significant scientific interest because of the potential insight it may give into the processes involved in the brain dysfunction of Alzheimer's."

'This gives all of us in Alzheimer research a tremendous new clue about new avenues of research.'

Recent work has demonstrated that TN F- $\alpha$, in addition to its proinflammatory functions, is a gliotransmitter and is responsible for regulation of synaptic function in neural networks.

R esearchers suggest that the findings yielded from etanercept administration may result from the drug targeting the effects of T N F- $\alpha$ on the synaptic mechanisms in Alzheimer's disease, and thus may demonstrate a potential area for future therapeutic endeavors.

'This is promising and innovative research, but in the early stages, and further work is needed before we can conclude etanercept could work as a treatment for Alzheimer's.'

Lead author of the study Edward Tobinick, of the U niversity of California, CA, USA, and Director of the Institutefor $N$ eurological Research, said the drug had "a very rapid effect that's never been reported in a human being before." $\mathrm{He}$ added, "It makes practical changes that are significant and perceptible, making a difference to his daily living."

Rebecca Wood, of the Alzheimer's Research Trust, commented, "This is promising and innovative research, but in the early stages, and further work is needed before we can conclude etanercept could work as a treatment for Alzheimer's."

Wood cautions that there is still a need for further studies, "We need to investigate whether it is safe and works in a larger number of patients as well as monitor the long-term effects. Scientists also need to check the benefits weren't just due to the placebo effect and establish whether any benefit is just temporary or whether the disease itself is slowed."

'Scientists a lso need to check the benefits weren't just due to the placebo effect and establish whether any benefit is just temporary or whether the disea se itself is slowed.'

N eil H unt, of the Alzheimer's society, further champions the view that more research must be carried out "... before any conclusions are drawn on TN F- $\alpha$ and the development of Alzheimer's disease."

Sources: Tobinick EL, Gross H : Rapid cognitive improvement in Alzheimer's disease following perispinal etanercept administration. J. N euroinflammation 5(1), 2 (2008); and,

Griffin W S: Perispinal etanercept: potential as an Alzheimer therapeutic. J. N euroinflammation 5(1), 3 (2008). 


\section{Peptide therapy inhibiting N F- $\kappa$ B may prevent Parkinson's disease progression}

Parkinson's disease (PD), a disease of old age with a prevalence of one in 100 in people over 60 years of age, is a slow neurodegenerative disease that affects neurons in the substantia nigra. The cause is unknown, with both the environment and genetics being implicated. The degeneration of neurons results in lower levels of the neurotransmitter dopamine, which leads to the characteristic symptoms of PD .

A recent study by Kali Pahan (Rush University M edical Centre, IL, USA) and colleagues, reports that peptide therapy may prevent PD progression. At present, treating neurological disorders with proteins, peptides and genes can be risky and painful, since they cannot cross the $B B B$ and so have to be injected into the brain. The current study reports on a peptide that is administered by intraperitoneal injection. "T his could be a new approach to halt disease progression in PD patients," says Pahan.

\section{'This could be a new approach to halt disea se progression in PD patients.'}

The peptide reverses the biochemical, cellular and anatomical changes of PD in a mouse model by blocking N F- $\mathrm{KB}$. The level of $\mathrm{N} \mathrm{F- \kappa B}$ is increased in the area of the midbrain that is affected in PD. Peptides, proteins and certain drugs cannot enter the brain because they cannot cross the BBB. "To overcome this problem, we have added a tag in front of that peptide that is helping the peptide enter into the brain," explains Pahan.

The peptide was shown to inhibit $\mathrm{NF}-\mathrm{\kappa B}$ and associated toxic molecules, protect neurons, normalize levels of neurotransmitters and improve motor function in the mouse model of PD, "N ow we need to translate this finding to the clinic and test this peptide in patients with PD . If these results can be replicated in PD patients, it would be a remarkable advance in the treatment of this devastating neurodegenerative disease," Pahan said.

Source: Ghosh A, Roy A, Liu X et al.: Selective inhibition of $N F-\kappa B$ activation prevents dopaminergic neuronal loss in a mouse model of Parkinson's disease. Proc. N atl Acad. Sci. U SA 104(47), 18754-18759 (2007).

\section{Evidence suggests stroke recovery depends on metabolic stress levels}

$\mathrm{H}$ igh-density lipoprotein (H DL), homocysteine and diabetes have been linked to poorer cognitive function and greater disability after stroke.

‘...HDL, homocysteine and diabetes have been linked to poorercognitive function and ... disa bility a fter stroke.'

This supports the view that metabolic stress plays a significant role in the post-stroke period.

These conclusions were made by researchers from the USA and were published in the $\mathrm{N}$ ovember 27 issue of N eurology. The results were drawn using data from the Vitamin Intervention for Stroke Prevention (VISP) trial using a linear mixed-effects model. A total of 3680 subjects were analyzed, all over
35 years of age, and entered into the VISP database between 1996 and 2003. Cognitive and motor function during a 2-year post-stroke period were assessed using scores from the M ini-M ental State Examination and modified Rankin Scale. Subjects with lower levels of both H D L and homocysteine were more likely to have memory difficulties and reduced motor activity following a stroke episode.

The aim of the study was, "To delineate factors associated with cognitive function following stroke and test the hypothesis that vascular risk factors associated with oxidative stress impair recovery."

In addition to the metabolic stress factors, increased age, recurrent stroke and left hemisphere cortical lesions were independent predictors of less successful cognitive recovery.
'Subjects with lower levels of both HDLand homocysteine were more likely to have memory difficulties and reduced motor activity following a stroke episode.'

G eorge N ewman, who worked on the study, commented that, "It's currently unclear why these factors are contributing to a slower stroke recovery and more research is needed. We need to focus our attention on identifying and treating these vascular risk factors."

Source: N ewman GC, Bang H , H ussain SI, Toole JF: Association of diabetes, homocysteine and $H D L$ with cognition and disability after stroke. N eurology 69, 2054-2062 (2007). 


\section{Research suggests breast cancer risk is not related to use of statins}

Previousfindings have demonstrated that statins areableto inhibit the proliferation of breast cancer cells both in vitro and in vivo in rodents, suggesting that they may be effective as a preventative approach to breast cancer. H owever, results from some trials contradict these findings, providing evidence that statins may, in fact, increase the risk of breast cancer.

Study investigators conducted structured telephone interviews to examine cases of incident breast cancer, diagnosed between 1995 and 2001, in 4179 women aged 50 years or older, as well as 4983 age-matched controls with no history of breast cancer.
In the 6 months prior to the trial, $7 \%$ of women had been prescribed a statin, comprising 271 women who had recovered from breast cancer and 336 women from the control group. Researchers found that statin use was not associated with risk of breast cancer, including amongst those women who had used statins for 10 years or more.

U se of hydrophilic statins was linked to a nonsignificant increase of breast cancer risk, whilst treatment with lipophilic statins had no effect on the risk of breast cancer. Breast cancer risk was not affected by duration of use of either hydrophilic or lipophilic statins.
'Breast cancer risk was not affected by duration of use of either hydrophilic or lipophilic statins.'

This study provides promising results concerning the risks of statins; however, researchers warn that further studies are required for confirmation of their findings.

Source: Pocobelli G, N ewcomb PA, Trentham-D ietz A, Titus-Ernstoff $L$, $H$ ampton JM , Egan KM: Statin use and risk of breast cancer. Cancer (2007) (Epub ahead of print).

\section{Faster assessment of patients suffering T IAs may prevent hundreds of strokes}

Transient ischemic attacks (TIAs), or mini strokes, are characterized by a temporary weakening of one side of the face and the corresponding arm. TIAs have al so been shown to dramatically increase the chance of suffering a major stroke within days of the onset of symptoms. In fact, some studies have calculated the risk to be as high as a one in four probability.

Early assessment of patients suffering TIAs is of utmost importance, and “... current UK guidelines recommend that all people who have had a TIA should be assessed by a specialist within 7 days of the start of symptoms," says $C$ raig Smith (U niversity of $M$ anchester, UK). H owever, a recent study by Smith and colleagues found that approximately two-thirds of patients who attended 'rapid access' TIA clinics were not seen within the recommended 7 days. In fact, in some cases access to the specialist clinics took twice as long as the guidelines recommend. " $O$ ur findings suggest that this standard is not being met and, in reality, TIA patients should ideally be assessed for risk of further stroke within a couple of days, if not on the same day as the initial symptoms," says Smith.

‘...TA patients should ideally be a ssessed for risk of further stroke within a couple of days, if not on the same day as the initial symptoms.'

The study monitored 711 people who had suffered a TIA or minor stroke 15 days earlier and who attended five centers in $M$ anchester and Liverpool. The risk of recurrent TIA, stroke, heart attack or death was monitored over a 3-month period and the $A B C D 2$ scoring system was used to detect the risk of stroke, even though specialist assessment was delayed.

A total of 25 peoplewent on to suffer a stroke, 100 patients suffered a further TIA during the 3-month follow-up and three people died. Commenting on these results, Smith said, "... this rate of stroke was relatively low due to the delay in being able to assess the patients after their initial TIA. Some studies have put the number of people suffering a major stroke within a week of a TIA as high as $10 \%$, which suggests even the 7-day guideline figure may be inadequate."

A number of factors contribute to the delay in assessment of TIA patients. An important factor is the patient not realizing the seriousness of their symptoms. The initial symptoms are temporary, lasting from minutesto hours, and thus patients often feel better by the time they are seen by a clinician.

The researchers conclude that "... if the speed with which TIA patients can be evaluated is improved, many strokes in the UK each year could be prevented."

Source: Selvarajah JR, Smith CJ, H ulmeS, Georgiou RF, Vail A, Tyrrell PJ: Prognosis in patients with transient ischemic attack (TIA) and minor stroke attending TIA services in the N orth West of England: the N ORTH STAR Study.

J. N eurol. N eurosurg. Psychiatry D O :10.1136/jnnp.2007.129163 (2007) (Epub ahead of print). 


\section{Progesterone shown to have mixed effects on Alzheimer's disease neuropathology}

A recent study has investigated the effect of progesterone on the development of Alzheimer's disease (AD). Although the falling estrogen levels in postmenopausal women are currently considered as a contributing factor to $A D$, the effect of progesterone has not been thoroughly investigated.

The researchers, from the $U$ niversities of California and Southern California, CA, USA, used a murine model to look at the effects of progesterone on $A D$-like neuropathology, both alone and in combination with estrogen. Triple transgenic mice were predisposed to develop AD. The progression of AD was then charted by analyzing $\beta$-amyloid accumulation, tau hyperphosphorylation and hippocampal-dependent behavioral impairments; all signs of AD .

The transgenic mice with intact ovaries showed signs of AD within
3 months of age, which worsened in the following 9 months; reflecting their transgenic status. Also, as expected, ovarectomized transgenic mice had significantly increased b-amyloid accumulation and reduced memory performance. Treating the ovarectomized mice with estrogen alone prevented the AD -like neuropathology. $H$ owever, when treated with estrogen and progesterone, the ovarectomized mice showed no improvement on

$\beta$-amyloid accumulation.

Unexpectedly, progesterone significantly reduced tau hyperphosphorylation, both alone and when administered in combination with estrogen. $H$ yperphosphorylation of the tau protein is a feature of $A D$, resulting in the transformation of tau from a soluble molecule into an insoluble paired helical filament. ‘...progesterone

signific a ntly reduced tau hyperphosphorylation, both

alone and when

administered in combination with estrogen.'

The authors concluded "T hese results demonstrate that estrogen and progesterone independently and interactively regulate $A D$-like neuropathology and suggest that an optimized hormone therapy may be useful in reducing the risk of $A D$ in postmenopausal women."

Source: Carroll JC, Rosario ER, Chang $L$ et al.: Progesterone and estrogen regulate Alzheimerlike neuropathology in female 3xT g-AD mice. J. N eurosci. 27(48), 13357-13365 (2007).

\section{Streamlined model may improve breast cancer risk-reduction strategies for postmenopausal women}

Estrogen receptor-positive breast cancer risk-reduction strategies require screening of large numbers of postmenopausal women. The current protocol for breast cancer risk assessment, the 'G ail model', has proven unpopular in the clinical environment, a fact attributed by the study authors, Chlebowski et al., to its perceived complexity. A recent survey adds weight to this claim with the finding that only $11 \%$ of primary-care providers had used the Gail model in the previous year.

A total of $25 \%$ of primary-care providers had prescribed antiestrogen therapy for risk reduction, although researchers believe many more women could benefit from this treatment and that the number may be increased by the use of a simpler assessment model. In order to instigate the efficacy of a simpler model, study investigators used data from 147,916 postmenopausal women enrolled in the Women's H ealth Initiative. The Gail model, as well as a more streamlined version of the model, was used to predict those most at risk from breast cancer. The variables included in the streamlined model were age, breast cancer in firstdegree relatives and previous breast biopsy examination.

The cohort was observed for 5 years and the accuracy of the models was assessed by observation of predicted and actual incident breast cancer. Results of the study demonstrated that the $G$ ail model and the streamlined model were able to predict those at risk of developing estrogen receptor-positive breast cancer at a similar level, with area under the curve values of 0.58 and 6.0 , respectively.

"For the first time, a postmenopausal woman can use a simple model and determine by herself if she is at increased risk of getting breast cancer. She could then raise this issue with her healthcare provider because interventions to reduce her risk of breast cancer are now available," concludes Chlebowski.

'For the first time, a
postmenopausal woman
can use a simple model and
determine by herself if she is
at increa sed risk of getting
breast cancer.'

Source: Chlebowski RT, Anderson GL, Lane DS et al.: Predicting risk of breast cancer in postmenopausal women by hormone receptor status. J. N atl Cancer Inst. 99(22), 1695-1705 (2007). 


\section{In brief...}

Range and severity of symptoms over time among older adults with chronic obstructive pulmonary disease and heart failure.

Walke LM, Byers AL, Tinetti ME, Dubin JA, M cCorkle R, Fried TR. Arch. Intern. Med. 167(22), 2503-2508 (2007).

An observational study was conducted amongst community-dwelling persons aged 60 years or above. Participants all suffered from chronic obstructive pulmonary disease (COPD) or heart failure (HF). Interviews were conducted a minimum of once every 4 months for a period of up to 2 years. Researchers ascertained the severity of seven different symptoms including physical discomfort, pain, fatigue, problems with appetite, depression, anxiety and limited activity by using the Edmonton Symptom Assessment Scale, which requires patients to rate their symptoms as absent, mild, moderate or severe.

At least $50 \%$ of COPD patients reported shortness of breath, physical discomfort, fatigue, and problems with appetite and anxiety, and at least $50 \%$ of HF patients reported physical discomfort, fatigue and appetite problems at the onset and conclusion of the study. The researchers conclude that the symptoms presented by COPD and HF in older persons represent a substantial burden to older persons and represent an area where improvement in symptom assessment and treatment is required.

\section{Usefulness of inflammatory and} haemostatic markers to predict short-term risk for death in middle-aged ischaemic stroke patients.

Rallidis LS, Vikelis M, Panagiotakos DB, Liakos GK, Krania E, Kremastinos DT: Acta Neurol. Scand. (2007) (Epub ahead of print).

Researchers recruited 231 acute ischemic stroke patients aged over 66 years. C-reactive protein (CRP), TNF- $\alpha$, fibrinogen and D-dimer levels were determined in the patients within $12 \mathrm{~h}$ of admission. A total of $6.5 \%$ of patients died whilst in hospital. Higher levels of CRP and fibrinogen in these patients were shown to be independently associated with death. D-dimer levels were also higher, but this association was negated when confounding factors were taken into account.

A $1 \mathrm{mg} / \mathrm{dl}$ increase in CRP was linked to a 20\% increase in the risk of dying and a $10 \mathrm{mg} / \mathrm{dl}$ increase in fibrinogen increased risk of dying by $18 \%$. Researchers conclude that the CRP and fibrinogen are independent predictors of early death in middle-aged ischaemic stroke patients, demonstrative of the importance of inflammation and coagulation in the evolution of ischemic stroke.

\section{"M y memory's not what it used to be." Study suggests why our brains deteriorate as we get older}

A recent study, published in the December 6 issue of $N$ euron has demonstrated that deteriorating neural connections are linked to normal cognitive decline. This decline was shown to occur even in the absence of serious pathologies such as Alzheimer's disease.

"O ur study now shows that cognitive decline in aging may be linked to disruption of communication between different regions of the brain," commented Randy Buckner, coauthor of the study. Using functional MRI, the researchers investigated the connectivity between frontal and posterior regions - associated with high-level cognitive functions - of the participants' brains. "The crosstalk between the different parts of the brain is like a conference call," explained Jessica Andrews-H anna, lead author of the study. "We were eavesdropping on this crosstalk and we looked at how activity in one region of the brain correlates with another."

‘...cognitive decline in

aging may be linked to distuption of

communication

between different

regions of the brain.'

A total of 93 healthy individuals, aged 18-93 years, were divided into two groups: young adults, aged 18-34 years $(n=38)$ and old adults aged 60-93 years $(n=55)$.
The researchers found that reduced correlation between the brain regions was associated with lower performance on cognitive tests.

"We found that in young adults, the front of the brain was pretty well in sync with the back of the brain," explained Andrews-H anna. "In older adults this was not the case. The regions became out of sync and they were less correlated with each other. Interestingly, the older adults with normal, high correlations performed better on cognitivetests."

The cause of this loss of synchrony was shown to be deterioration in the brain's white matter, resulting in disrupted connectivity between the brain regions.

"It may help explain why some people are just as sharp in their 90 s as they were in their 40s," commented Andrews-H anna. "We all age differently and cognitive abilities vary considerably among individuals."

The researchers acknowledge that more research is required, however, they are hopeful that their work will aid in our understanding of 'normal' aging, as Buckner pointed out. "We can use this new approach (correlating the activities of different regions of the brain) as a tool to understand variation between individuals. We can also explore risk factors for breakdowns (in these pathways), like cardiovascular health."

Source: Andrews-Hanna JR, Snyder AZ, Vincent $J L$ et al.: Disruption of large-scale brain systems in advanced aging. Neuron 56, 924-935 (2007).

\author{
About the Bulletin Board \\ If you have newsworthy information, please contact: \\ Shreeya Nanda, Commissioning Editor, \\ Aging Health, Future Medicine Ltd, Unitec House, 2 Albert Place, \\ London N3 1QB, UK \\ s.nanda@futuremedicine.com \\ Tel.: +44 (0)20 83716090 \\ Fax: $+44(0) 2083432313$
}

